\title{
Population Pharmacokinetic Analysis to Assist Dose Selection of the L-Ornithine Salt of Phenylacetic Acid
}

\author{
Xiaofeng Wang ${ }^{1} \cdot$ Regis A. Vilchez ${ }^{2}$
}

Accepted: 5 September 2021 / Published online: 17 November 2021

(c) The Author(s) 2021

\begin{abstract} removal of ammonia. weight $\leq 50 \mathrm{~kg}$ ) and severe hepatic impairment.

\section{Introduction}

Hepatic encephalopathy (HE) can arise in the setting of acute liver failure, chronic progressive liver disease in the context of advanced liver cirrhosis, and/or as a result of portocaval shunting with or without liver disease [1]. The pathogenesis of HE is incompletely understood, but the increase in venous ammonia levels remains central to our understanding [2]. Phenylacetic acid (PAA) is a well-characterized ammonia scavenger [3]. Formulations that contain PAA or phenylbutyrate as the primary active moiety include sodium phenyl-butyrate and glycerol phenylbutyrate and are approved for the acute and/or maintenance treatment of urea cycle disorders (UCD)
\end{abstract}

Background and Objective L-Ornithine phenylacetate is an intravenous formulation of the L-ornithine salt of phenylacetic acid under development for the treatment of hepatic encephalopathy. Very limited clinical data in patients are available, with a phase II study in target patients not designed for dose finding, to support phase III dose selection in a global development program. The objective of the present population pharmacokinetic modeling and simulation was to evaluate dose selection for target patient populations with a low body weight, ethnicity, and hepatic impairment in a global clinical study.

Methods A population pharmacokinetic model was developed based on plasma concentrations of L-ornithine, phenylacetic acid, and phenylacetylglutamine data from four clinical trials in healthy subjects and patients with stable cirrhosis or hospitalized adult patients with liver cirrhosis and hepatic encephalopathy. A covariate analysis was conducted to identify source of variability to support dose selection for global clinical development of L-ornithine phenylacetate. Phenylacetylglutamine formation in the pharmacokinetic model also quantified pharmacodynamic effects measured by ammonia removal.

Results Body weight and hepatic function were significant covariates determining phenylacetic acid exposure. After accounting for body weight, there was no difference between tested Caucasian and Asian populations in phenylacetic acid exposure. Renal dysfunction significantly reduced phenylacetylglutamine excretion. However, renal impairment had no impact on plasma phenylacetic acid and free ammonia levels. Exploratory modeling suggested that L-ornithine might enhance the

Conclusions With a flat dosing algorithm, special consideration must be given to patients with a small body size (i.e., body

Regis A. Vilchez

regis.vilchez@alumni.bcm.edu

1 Clinical Pharmacology and Pharmacometrics, Mallinckrodt Pharmaceuticals, Hampton, NJ, USA

2 Clinical Development, Mallinckrodt Pharmaceuticals, 53 Frontage Rd, Hampton, NJ 08827, USA

\section{Key Points}

To date, there are only two clinical studies of L-ornithine phenylacetate. One was a single ascending dose study in patients with stable cirrhosis, and the other was a phase II study in targeted patients with hepatic encephalopathy; however, it was not designed for dose finding. With very limited clinical data, dose selection for phase III studies in different regions was challenging.

The modeling and simulation provided information to support the rationale for the phase III dosing regimen that was accepted by the US Food and Drug Administration, the European Medicines Agency, and the Pharmaceuticals and Medical Devices Agency.

From a modeling methodology point of view, the work presented here also demonstrates the importance of simplifying a model without losing its validity. 


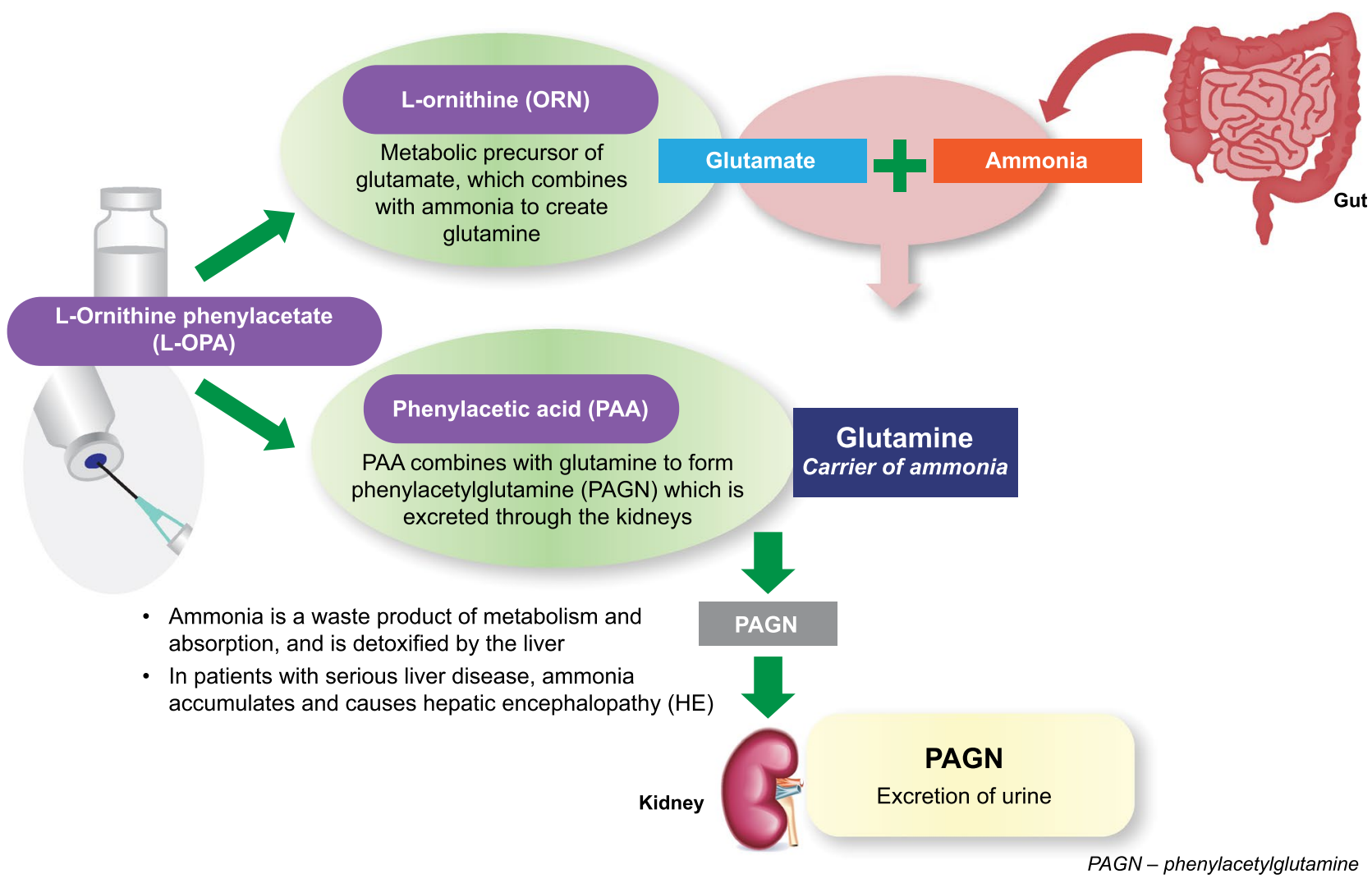

Fig. 1 Schematic of the mechanism of action of L-ornithine and phenylacetate

[4-6]. Body size (weight or body surface area [BSA]) has a significant impact on PAA exposure [7, 8]. The approved doses of sodium phenylbutyrate and glycerol phenylbutyrate are based on body weight or BSA [4-6]. In Japanese patients with UCD, sodium phenylbutyrate follows the same dosing algorithm as in Caucasian patients [9], suggesting that ethnicity does not play a role in the dosing regimen once body size is taken into consideration.

L-Ornithine phenylacetate (L-OPA) is an intravenously administered formulation of the L-ornithine (ORN) salt of PAA under development for the treatment of HE. Once administered, L-OPA dissociates to form free PAA and ORN. The metabolic pathway of L-OPA and its interaction with ammonia is illustrated in Fig. 1. Phenylacetic acid removes ammonia by conjugating irreversibly with glutamine (catalyzed by phenylacetyl coenzyme A:glutamine acyltransferase) in the liver to form phenylacetylglutamine (PAGN). Glutamine is formed when ammonia combines with glutamate. Because glutamine formation is a reversible reaction, the formation of PAGN prevents glutamine from releasing ammonia back into systemic circulation. One mole of PAGN formation removes 2 moles of ammonia. L-Ornithine is an endogenous non-proteogenic amino acid. Some fraction of ORN is converted to glutamate, which increases the glutamate level and may indirectly enhance the removal of ammonia [10]. As PAA is the primary active moiety and has been reported to be associated with neurotoxicity in patients with cancer at concentrations above $490 \mu \mathrm{g} / \mathrm{mL}$ [11], the focus of the present analysis was to identify and quantify the source of variability for PAA exposure.

The global clinical development program for L-OPA includes Japan and other countries in Asia. The target population is hospitalized adult patients with liver cirrhosis and hyperammonemia associated with an episode of HE. In the planned phase III clinical trial (ClinicalTrials. gov NCT04128462), the selected dosing regimen for Caucasian patients is $20 \mathrm{~g}$ infused over $6 \mathrm{~h}$ as a loading dose followed by $15 \mathrm{~g}$ infused over $18 \mathrm{~h}$ (day 1 ), and $15 \mathrm{~g}$ infused over $24 \mathrm{~h}$ each additional day (day 2 to day 5). This flat dose in the phase III study was not derived from population pharmacokinetic (PK) modeling, but was supported by the exposure and safety data from a phase II study in the same target patient population. In the phase II study, the dose was selected based on baseline calculation of hepatic synthetic and portal elements of the Child-Pugh (C-P) score (4-6 points, $20 \mathrm{~g} / 24 \mathrm{~h}$; 7-9 points, $15 \mathrm{~g} / 24 \mathrm{~h}$; and $10-12$ points, $10 \mathrm{~g} / 24 \mathrm{~h}$, all by continuous infusion) over 5 days of treatment. The C-P score is a four-item, 3-point (1-3) score that assesses ascites (none, mild/moderate, tense), total bilirubin 
$(<2 \mathrm{mg} / \mathrm{dL}[<34 \mu \mathrm{mol} / \mathrm{L}], 2-3 \mathrm{mg} / \mathrm{dL}[34-51 \mu \mathrm{mol} / \mathrm{L}]$, $>3 \mathrm{mg} / \mathrm{dL}[>51 \mu \mathrm{mol} / \mathrm{L}])$, albumin $(>3.5 \mathrm{~g} / \mathrm{dL}[>35 \mathrm{~g} / \mathrm{L}]$, $2.8-3.5 \mathrm{~g} / \mathrm{dL}[28-35 \mathrm{~g} / \mathrm{L}],<2.8 \mathrm{~g} / \mathrm{dL}[<28 \mathrm{~g} / \mathrm{L}])$, and international normalized ratio $(<1.7,1.7-2.3,>2.3)$ or, if not available, prothrombin time ( $<4,4-6,>6$ s prolonged). The C-P score was also calculated for an individual patient at screening. The $15 \mathrm{~g}$ with $24 \mathrm{~h}$ of infusion in patients with C-P B or C-P C appeared to achieve comparable clinical efficacy compared to the $20 \mathrm{~g}$ with $24 \mathrm{~h}$ of infusion in patients with C-P B. In addition, PAA exposure at $20 \mathrm{~g}$ in $\mathrm{C}-\mathrm{P}$ B was $36 \%$ higher than that in C-P C patients at $15 \mathrm{~g}$, and no difference in adverse events was observed across different dose levels [8, 12].

Patients with HE have hepatic dysfunction by definition, and some patients also have various degrees of renal impairment $[13,14]$. Asian populations generally have a lower body weight than Caucasian populations $[15,16]$. The combination of low body weight and severe hepatic impairment could elevate PAA exposure beyond the degree determined to have been safe in the phase II study [12], whose population was mostly Caucasian patients. The objective of the present analysis was to identify and quantify various covariates of patients with HE that significantly alter the exposure of PAA and PAGN using population PK modeling and simulations. The results would inform dose selection of L-OPA for patients with various degrees of hepatic or renal impairment, different ethnicities, or a wide range of body weight in the global clinical development program for L-OPA.

\section{Methods}

\subsection{Clinical Study Design and Data for Model Development}

Pharmacokinetic data from four clinical studies (protocols OCR002-HV201, OCR002-HE201, OCR002-HE209, and MNK61051112) were included in this analysis. An institutional review board approved each study protocol. All participants gave written informed consent prior to the initiation of study procedures. Detailed information about the study design and results is provided in separate publications $[8$, 12]. Brief information about the study designs is provided below.

HV201 (protocol OCR002-HV201) was a phase I, randomized, double-blind, placebo-controlled, single-ascending dose and multiple-ascending dose study in healthy subjects. The doses in the single-ascending dose part were 1 , $3,10,20$, and $30 \mathrm{~g}$ infused over $4 \mathrm{~h}$, and 30,40 , and $60 \mathrm{~g}$ infused over $24 \mathrm{~h}$. In the multiple-ascending dose part, 1, 3, 10, and $20 \mathrm{~g}$ were infused over $4 \mathrm{~h}$ for 5 days [17]. HE201 (protocol OCR002-HE201) was a phase IIa, randomized, double-blind, placebo-controlled, single-ascending dose study conducted in patients with stable cirrhosis to evaluate the tolerability and PK of L-OPA. Tested doses were $1,3,10,20$, and $40 \mathrm{~g}$ infused over $4 \mathrm{~h}$, and 10, 20, and $40 \mathrm{~g}$ infused over $24 \mathrm{~h}[17,18]$. HE209 (ClinicalTrials.gov NCT01966419; protocol OCR002-HE209) was a phase IIb, placebo-controlled, randomized, double-blind clinical study to evaluate the safety, pharmacokinetics, and efficacy of L-OPA in hospitalized patients with liver cirrhosis and an acute episode of HE. Study design and results of HE209 were described by Rahimi and colleagues [12]. The dosage of L-OPA in study HE209 was based on a baseline calculation of the hepatic synthetic and portal elements score (4-6 points, $20 \mathrm{~g} / 24 \mathrm{~h}$; 7-9 points, $15 \mathrm{~g} / 24 \mathrm{~h}$; and 10-12 points, $10 \mathrm{~g} / 24 \mathrm{~h}$, all by continuous infusion) over 5 days of treatment. MNK61051112 (protocol MNK61051112) was an open-label, parallel, phase I study that assessed the PK and safety of L-OPA in healthy Chinese Han and Japanese subjects. The dose selected for the study was $20 \mathrm{~g}$ infused over $24 \mathrm{~h}$.

Rich plasma PK samples were collected from all studies except study HE209, in which pre-dose samples were collected on each dosing day, and additional samples were collected at three time points after the end of the last infusion (Table S1 of the Electronic Supplementary Material [ESM]). Plasma concentrations of ORN, PAA, and PAGN were measured using a validated liquid chromatographytandem mass spectrometry method [19]. The lower limits of quantification are $10.00 \mu \mathrm{g} / \mathrm{mL}$ for ORN and PAA, and $5.00 \mu \mathrm{g} / \mathrm{mL}$ for PAGN. The inter-assay precision (expressed as \%CV) ranged from 3.4 to $6.2 \%$ for PAA, from 3.2 to $6.1 \%$ for PAGN, and from 2.8 to $5.8 \%$ for ORN in the concentration range up to $1000 \mu \mathrm{g} / \mathrm{mL}$. Interassay accuracy (expressed as \% relative error) ranged from -1.3 to $4.0 \%$ for PAA, from -1.3 to $4.5 \%$ for PAGN, and from 0.1 to $6.5 \%$ for ORN in the concentration range up to $1000 \mu \mathrm{g} / \mathrm{mL}$.

\subsection{Population PK Model Development}

Plasma concentrations of ORN, PAA, and PAGN from studies mentioned above were included in the development of the population PK model. The population PK model was developed using nonlinear mixed-effects modeling with NONMEM (version 7.4, Icon Development Solutions, Ellicott City, MD, USA). The first-order conditional estimation method with interaction was used for parameter estimation. Data summary, plotting, and model diagnostics were completed with $\mathrm{R}$, version 3.5.2. Model-based simulation was also conducted using NONMEM.

A semi-mechanistic model was explored to describe the PK profiles of ORN, PAA, ammonia, and glutamine, as 


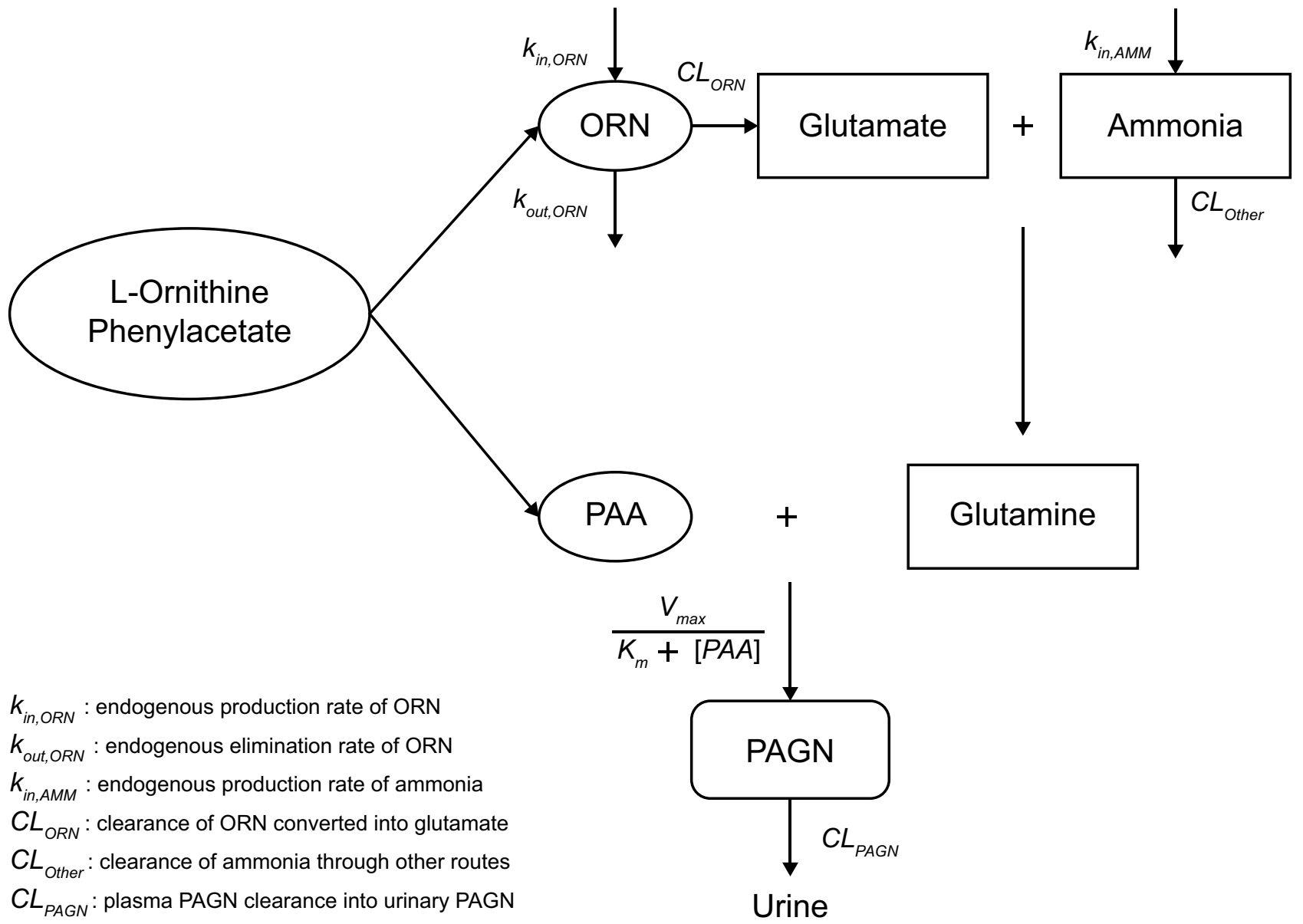

Fig. 2 Schematic of the structural model for ornithine phenylacetate. $K_{m}$ Michaelis constant, $O R N$ ornithine, $P A A$ phenylacetic acid, $P A G N$ phenylacetylglutamine, $V_{\max }$ maximum rate

well as the formation of PAGN (Fig. 2). Equations (1-6) describe the model shown in Fig. 2:

$V_{\mathrm{ORN}} \frac{\mathrm{d} C_{\mathrm{ORN}}}{\mathrm{d} t}=k \mathrm{in}_{\mathrm{ORN}}-k \mathrm{out}_{\mathrm{ORN}} C_{\mathrm{ORN}}+$ Rate $_{\mathrm{ORN}-\mathrm{inf}}-\mathrm{CL}_{\mathrm{ORN}} C_{\mathrm{ORN}}$,

$V_{\mathrm{PAA}} \frac{\mathrm{d} C_{\mathrm{PAA}}}{\mathrm{d} t}=$ Rate $_{\mathrm{PAA} \_ \text {inf }}-f\left(k_{34}, c_{\mathrm{PAA}}, C_{\mathrm{Glutamine} . . .}\right)$,

$V_{\mathrm{PAGN}} \frac{\mathrm{d} C_{\mathrm{PAGN}}}{\mathrm{d} t}=f\left(k_{34}, c_{\mathrm{PAA}}, C_{\mathrm{Glutamine} . .}\right)-\mathrm{CL}_{\mathrm{PAGN}} C_{\mathrm{PAGN}}$,

$V_{\text {Glutamate }} \frac{\mathrm{d} C_{\mathrm{Glutamate}}}{\mathrm{d} t}=k \mathrm{in}_{\mathrm{Glutamate}}-k$ out $_{\text {Glutamate }} C_{\text {Glutamate }}$

$-f\left(k_{12}, 2 C_{\text {Amm }}, C_{\text {Gluamate }}, \ldots\right)+f\left(k_{21}, C_{\text {Glumine }}, \ldots\right)$,

$V_{\text {Glutamine }} \frac{\mathrm{d} C_{\text {Glutamine }}}{\mathrm{d} t}=f\left(k_{12}, 2 C_{\text {Amm }}, C_{\text {Gluamate }}, \ldots\right)$ $-f\left(k_{21}, C_{\text {Glumine }}, \ldots\right)-f\left(k_{34}, c_{\mathrm{PAA}}, C_{\text {Glutamine }}, \ldots\right)$,

$$
\begin{aligned}
V_{\mathrm{AMM}} \frac{\mathrm{d} C_{\mathrm{Amm}}}{\mathrm{d} t}= & k \operatorname{in}_{\mathrm{Amm}}-\mathrm{CL}_{\mathrm{Amm}} C_{\mathrm{Amm}} \\
& -f\left(k_{12}, 2 C_{\text {Amm }}, C_{\text {Gluamate }}, \ldots\right) \\
& +2 f\left(k_{21}, C_{\text {Glumine }}, \ldots\right) .
\end{aligned}
$$

$K$ in is the apparent zero-order production rate and $k$ out is the first-order rate constant (unit as L/h) for the elimination for endogenous reactants; $V$ and CL are volume of distribution and clearance, respectively; $C$ represents plasma concentration in molar units; $k$ is the rate constant for elimination or biotransformation; and Rate ${ }_{\text {inf }}$ is the infusion rate of ORN and PAA when L-OPA was administered. Subscripts represent the reactants (Amm is for ammonia) or different reaction-rate constants.

In Eqs. (1-6), a general function, $f(k$, reactants, ...), was used to describe the rate of biotransformation. Those functions can be linear or nonlinear. For example, if the reaction of PAA and glutamine follows the first order on 
both PAA and glutamine, the rate for PAGN formation can be expressed as $k_{34} C_{\mathrm{PAA}} C_{\mathrm{Glutamine}}$. Each mole of glutamine formation consumes 2 moles of ammonia. Therefore, molar unit was used in the equations. In Eq. (2), the production and elimination of the endogenous PAA was omitted, as PAA exists in the human body at a very low level (pre-dose PAA was below the quantifiable level). The elimination of PAA following the administration of L-OPA is also a function of glutamine, which was affected by glutamate level and ORN, etc. The biotransformation of PAA to form PAGN was assumed to be the only route of PAA clearance. The production of glutamate was believed to be increased due to ORN administration. For ammonia, the production and removal of ammonia in patients are disease and individual dependent.

Initial conditions for Eqs. (1-6) were given as below:

For ORN, $k \mathrm{in}_{\text {orn }}=k_{\text {out }}$ orn $_{\text {orn_baseline }}$ was derived assuming that ORN is at a stable level prior to L-OPA administration, that is, $\frac{\mathrm{d} C_{\text {orm }}}{\mathrm{d} t}=0$. For all other reactants, the concentrations at time $=0$ were at the baseline levels, respectively.

This nonlinear model with a large number of parameters and a lack of quantitative information on (1) glutamate levels and (2) the production and elimination of endogenous ORN and ammonia was not only computationally intensive but also made identification of unique parameter values difficult (unpublished data, Mallinckrodt Pharmaceuticals). Therefore, every effort was made to simplify the structural model during model development.

The first simplification made was to describe the PK of ORN using a one-compartment linear model with the endogenous level incorporated in the model as the baseline level instead of using an indirect response model describing the endogenous ORN. The rationale for this simplification was based on the observed, rather stable, plasma ORN levels in the placebo arm during 5 days of treatment, where ORN levels were maintained at the baseline [20]. The second simplification was made by assuming that the transition of PAA to PAGN followed Michaelis-Menten kinetics and was the only biotransformation responsible for PAA elimination [7]. This biotransformation also quantified the formation of PAGN and the removal of ammonia, as 1 mole of PAA and 1 mole glutamine forms 1 mole of PAGN and 1 mole glutamine includes 2 moles of ammonia. The third simplification was made by assuming that the enhancement of ammonia removal due to the increase of the glutamate pool from exogenous ORN was reflected in the maximum rate $\left(V_{\max }\right)$ of the Michaelis-Menten kinetics. Thus, PAA plasma concentration profiles were described by a one-compartment model with Michaelis-Menten kinetics for elimination, and PAGN plasma concentrations were described by a single compartment with the input rate equal to the rate of elimination of PAA. Molar units were used for all concentrations in the model. Detailed information on model simplification is provided in the ESM.

Sources of variability were explored through a covariate analysis. Covariates tested included age, sex, body weight, ethnicity, and study. Additional covariates such as renal and hepatic function were evaluated with PK data from the patient population (Table S2 of the ESM). Evaluation of covariates on the PK parameters of L-OPA depended on clinical relevance, changes in the objective function, reduction in between-subject variability, and the improvement of the fitting. The effects of ethnicity on PK were assessed using PK data from studies on healthy Caucasian and Chinese/Japanese subjects. During this step, the focus was on the ethnic factor contributing to PAA PK because PAA plasma concentrations above $490 \mu \mathrm{g} / \mathrm{mL}$ have been reported to be associated with reversible neurological adverse events in patients with cancer who were receiving PAA intravenously [11].

Between-subject variability for a PK parameter was assumed to follow a log normal distribution, and the intra-individual variability was described by both a proportional and an additive error combination. Model evaluation was conducted using standard diagnostic tools such as goodness-of-fit criteria (e.g., objective function value difference), diagnostic plots, and visual predictive checks. Bootstrapping was conducted when covariance steps failed.

\subsection{Simulation to Support Dose Selection for Asian Patients}

Model-based simulations were conducted to support dose selection in Asian patients with HE. As the PAA plasma concentration was of interest, simulations were focused on PAA exposure in an Asian patient population. For comparison, 500 virtual Caucasian patients with HE per C-P group C-P A, C-P $\mathrm{B}$, and $\mathrm{C}-\mathrm{P} \mathrm{C}$, were generated. The body weight distribution of those virtual Caucasian patients was similar to that of patients in study HE209, which is also the targeted patient population of the phase III clinical study. The dosing regimen for the simulation was the same as for the planned phase III study, namely $20 \mathrm{~g}$ infused over $6 \mathrm{~h}$ (loading dose) followed by $15 \mathrm{~g}$ infused over $18 \mathrm{~h}$ and $15 \mathrm{~g}$ infused over $24 \mathrm{~h}$ on the remaining days 2-5. Virtual Asian patients with HE (C-P A/B/C) were generated assuming that the mean value of the body weight was $20 \%$ less than that of Caucasian patients. The same dosing regimen as for Caucasian patients was selected for the simulations. Dose reduction for Asian patients was considered to ensure the predicted PAA exposure would be comparable to that of Caucasian patients. The population PK model from the patient population was used for simulations of PAA exposure in Caucasian patients. When using the same model to simulate PAA exposure in an Asian patient population, any ethnic factors identified during model development were incorporated into the simulations. 


\section{Results}

\subsection{Population PK Model}

Table 1 presents demographic and disease information of individuals included in the PK model development. Hepatic function was assessed by $\mathrm{C}-\mathrm{P}$ score and renal function was assessed by creatinine clearance. Among the patient population, $21 \%$ of the participants were classified as C-P A, 31\% as C-P B, and $48 \%$ as C-P C; $51 \%$ of patients had normal renal function, and $27,20,2 \%$ had mild, moderate, and severe renal impairment, respectively. The lowest creatinine clearance was $26 \mathrm{~mL} / \mathrm{min}(n=2)$.

Mathematical equations for the simplified structural population PK model from the semi-mechanistic model shown in Fig. 2 are given here:

$$
\begin{aligned}
& \frac{\mathrm{d} A_{1}}{\mathrm{~d} t}=\text { Rate }_{\mathrm{inf}}-\mathrm{CL}_{\mathrm{ORN}} \frac{A_{1}}{V_{\mathrm{ORN}}}, \\
& \frac{\mathrm{d} A_{2}}{\mathrm{~d} t}=\text { Rate }_{\mathrm{inf}}-\frac{V_{\mathrm{max}} A_{2} / V_{\mathrm{PAA}}}{K_{\mathrm{m}}+A_{2} / V_{\mathrm{PAA}}},
\end{aligned}
$$

$\frac{\mathrm{d} A_{3}}{\mathrm{~d} t}=\frac{V_{\mathrm{max}} A_{2} / V_{\mathrm{PAA}}}{K_{\mathrm{m}}+A_{2} / V_{\mathrm{PAA}}}-\mathrm{CL}_{\mathrm{PAGN}} \frac{A_{3}}{V_{\mathrm{PAGN}}}$

$A_{1}$ represents the exogenous amount of ORN; $A_{2}$ and $A_{3}$ represent the amount of PAA and PAGN, respectively. $\mathrm{CL}$ and $V$ represent clearance and volume of distribution, respectively, for each moiety. $K_{\mathrm{m}}$ is the Michaelis-Menten constant. Additional effort was made to explore the enhancement with the exogenous ORN from the administration of L-OPA. For example, $V_{\max }$ included a factor of $(1+$ Coe $\left.\times A_{1} / V_{\text {ORN }}\right)$, where Coe $\times A_{1} / V_{\text {ORN }}$ is the contribution to $V_{\max }$ due to the administration of L-OPA. Results from the covariate analysis are presented in the next sections.

\subsection{ORN in Healthy Subjects and Patients}

The model provided above adequately described the baseline-adjusted concentration of ORN in healthy subjects (Figs. S1 and S2 of the ESM). Covariate testing suggested that ethnicity (Caucasian vs Asian) has a significant impact on the PK of ORN. No statistically significant difference in ORN PK was observed between Chinese and Japanese individuals, although the sample size was small. A difference in

\begin{tabular}{|c|c|c|c|c|}
\hline & Sex & $N$ & $\begin{array}{l}\text { Age, median } \\
\text { Mean (SD), years }\end{array}$ & $\begin{array}{l}\text { Weight, median } \\
\text { Mean (SD), kg }\end{array}$ \\
\hline \multicolumn{5}{|c|}{ Healthy subject population $(N=46)$} \\
\hline \multirow[t]{2}{*}{ Caucasian } & Female & 22 & $\begin{array}{l}24.5 \\
23.3(12.8)\end{array}$ & $\begin{array}{l}66.5 \\
68.9(8.0)\end{array}$ \\
\hline & Male & 7 & $\begin{array}{l}21.0 \\
20.9(1.6)\end{array}$ & $\begin{array}{l}73.2 \\
71.0(5.6)\end{array}$ \\
\hline \multirow[t]{2}{*}{ Japanese } & Female & 6 & $\begin{array}{l}42.0 \\
45.0(12.2)\end{array}$ & $\begin{array}{l}57.0 \\
60.8(12.0)\end{array}$ \\
\hline & Male & 2 & $\begin{array}{l}44.0 \\
44.0(12.7)\end{array}$ & $\begin{array}{l}79.1 \\
79.1(5.2)\end{array}$ \\
\hline Chinese & Male & 8 & $\begin{array}{l}45.5 \\
39.4(9.1)\end{array}$ & $\begin{array}{l}74.2 \\
75.8(8.4)\end{array}$ \\
\hline Asian & Male & 1 & 26.0 & 72.0 \\
\hline \multicolumn{5}{|c|}{ Patient population $(N=152)$} \\
\hline \multirow[t]{4}{*}{$\begin{array}{l}\text { Caucasian or unknown } \\
\text { ethnicity }\end{array}$} & Female & 59 & $\begin{array}{l}59.0 \\
58.0(9.8)\end{array}$ & $\begin{array}{l}72.6 \\
73.9(18.0)\end{array}$ \\
\hline & Male & 93 & $\begin{array}{l}57.0 \\
56.4(9.5)\end{array}$ & $\begin{array}{l}84.5 \\
86.3(20.1)\end{array}$ \\
\hline & Renal fu & d by creatinine cle & & \\
\hline & Normal & Mild impairment & Moderate impairment & Severe impairment \\
\hline \multirow[t]{3}{*}{ Patients, $n(\%)$} & $78(51)$ & $41(27)$ & $30(20)$ & $3(2)$ \\
\hline & & \multicolumn{3}{|l|}{ Hepatic function } \\
\hline & & Child-Pugh A & Child-Pugh B & Child-Pugh C \\
\hline Patients, $n(\%)$ & & $32(21)$ & $47(31)$ & $73(48)$ \\
\hline
\end{tabular}

Table 1 Ethnicity, demographics, and disease characteristics

$S D$ standard deviation 
ORN PK was observed between age groups (approximately $\leq 36$ vs $>36$ years of age).

The same PK model from healthy subjects was applied to the ORN concentrations in patients with stable cirrhosis or cirrhosis with HE (Figs. S3 and S4 of the ESM). The covariate analysis revealed that sex, hepatic dysfunction assessed by C-P category, weight (range $45-153 \mathrm{~kg}$ ), and renal function have a significant impact on the PK of ORN. For example, $\mathrm{CL}_{\mathrm{ORN}}$ was proportional to the body weight ${ }^{0.82}$ and to $\mathrm{CLcr}^{0.61}$. In addition, the reduction in $\mathrm{CL}_{\mathrm{ORN}}$ from patients with C-P B or C-P C from C-P A was $37 \%$ or $55 \%$, respectively. The impact of age on ORN PK that was observed in healthy subjects could not be confirmed in patients. It was likely due to the fact that only six patients were $\leq 36$ years of age among 152 participants in the patient PK population dataset (defined as all patients whose PK profiles contained at least one post-dose data point with a quantifiable concentration value) [Figs. S3-S4 of the ESM].

\subsection{PAA in Healthy Subjects to Identify any Ethnic Factor}

The simplified model described the observed PAA plasma concentrations well across all dose levels with a range of 10-60 g (4- or 24-h infusion), while substantially reducing computation intensity. The covariate analysis suggested that body weight had a significant impact on $V_{\max }$ in the Michaelis-Menten equation for PAA elimination. Sex was a statistically significant covariate only for $V_{\mathrm{PAA}}$. Because steady-state concentration following constant infusion only depends on the rate of input and elimination, sex will not affect PAA concentration at steady state. Once body weight and sex were introduced into the covariate model, the impact of ethnicity (Caucasian vs Japanese or Chinese) on the PK parameters of PAA was no longer significant. However, given the lower body weight in the Asian patient population, higher PAA exposure is likely to be observed. Diagnostic plots of the final model in healthy subjects are provided in Fig. S5 of the ESM.

\subsection{PAA and PAGN in Patients}

The same structural model for healthy subjects also accurately described the PK profiles of PAA from studies HE201 and HE209. Covariate testing suggested that body weight and liver function contributed significantly to PAA exposure through elimination. Body weight was also a significant covariate for $V_{\mathrm{PAA}}$; however, the effect of sex on the volume of distribution was not significant compared with the healthy volunteer population. Results of the final model for patients are presented in Table 2. Model evaluation through diagnostic plots is presented in Fig. 3A, B. A visual predictive check is presented in Fig. 4.
Table 2 presents the PK parameters derived from the population PK modeling. The maximum elimination rate for PAA was nearly proportional to body weight. The other covariate that had a significant impact on PAA exposure was hepatic function. Compared with C-P A patients, $V_{\max }$ in patients with C-P B and C-P C was reduced by $37 \%$ and $61 \%$, respectively. This derivation agreed with the observed PAA concentration at steady state in study HE209, where the median steady-state PAA concentration for C-P C was approximately $36 \%$ higher than for C-P B at the $15 \mathrm{~g} / 24 \mathrm{~h}$ infusion. Renal impairment had no impact on exposure to PAA. However, renal function is a significant covariate correlated with clearance of PAGN, with $\mathrm{CL}_{\mathrm{PAGN}}$ proportional to $\mathrm{CLCr}^{0.8}$. The estimated typical value of the apparent clearance for PAGN representing the population with normal renal function was $14.9 \mathrm{~L} / \mathrm{h}$ and volume of distribution was $33.2 \mathrm{~L}$.

\subsection{Simulated PAA Exposure in Asian Patients with HE}

Simulated PAA PK profiles (median, 5th and 95th percentiles) in Caucasian and Asian patients with $\mathrm{HE}$ at the

Table 2 Final population pharmacokinetic model parameters for PAA in patients

\begin{tabular}{|c|c|c|c|}
\hline Parameters & Typical value & Standard error & $\begin{array}{l}\text { Values } \\
\text { converted to } \\
\mu \mathrm{g} / \mathrm{mL}\end{array}$ \\
\hline$V_{\max }, \mathrm{mmol} / \mathrm{h}$ & 12.4 & 1.94 & - \\
\hline$K_{\mathrm{m}}, \mathrm{mM}$ & 1.33 & 0.248 & 179 \\
\hline$V_{\mathrm{PAA}}, \mathrm{L}$ & 24.4 & 4.25 & - \\
\hline Weight power ${ }^{V \max }$ & 0.97 & 0.202 & - \\
\hline Weight power ${ }^{\text {VPAA }}$ & 0.79 & 0.178 & - \\
\hline \multicolumn{4}{|l|}{$V_{\max }$ ratio } \\
\hline $\mathrm{C}-\mathrm{P} \mathrm{B} / \mathrm{C}-\mathrm{P} \mathrm{A}$ & 0.63 & 0.0972 & - \\
\hline $\mathrm{C}-\mathrm{P}$ C/C-P A & 0.39 & 0.0539 & - \\
\hline \multicolumn{4}{|l|}{$\mathrm{V}_{\mathrm{PAA}}$ ratio } \\
\hline $\mathrm{C}-\mathrm{P} \mathrm{BC} / \mathrm{C}-\mathrm{P} \mathrm{A}$ & 1.89 & 0.328 & - \\
\hline \multicolumn{4}{|c|}{ Inter-individual variability } \\
\hline$V_{\max }, \%$ & 48 & 0.118 & - \\
\hline$K_{\mathrm{m}}, \%$ & 92 & 0.370 & - \\
\hline$V_{\mathrm{PAA}}, \%$ & 41 & 0.0272 & - \\
\hline \multicolumn{4}{|l|}{ Error model } \\
\hline $\begin{array}{l}\text { PAA proportional } \\
\text { error, } \%\end{array}$ & 35 & - & - \\
\hline PAA additive error & - & - & 2.23 \\
\hline
\end{tabular}

$C-P A / B / C$ Child-Pugh class A, B, or C, $K_{m}$ Michaelis-Menten constant, $P A A$ phenylacetic acid, $V_{\max }$ maximum rate, $V_{P A A}$ volume of distribution of PAA 
Fig. 3 A Final model diagnostic plots for phenylacetic acid in patients. CWRES conditional weighted residuals, $D V$ observed data values, IPRED individual predictions, $P R E D$ population predictions. B Final model of conditional weighted residuals (CWRES) distribution and quantile-quantile (Q-Q) plot for phenylacetic acid in patients
A
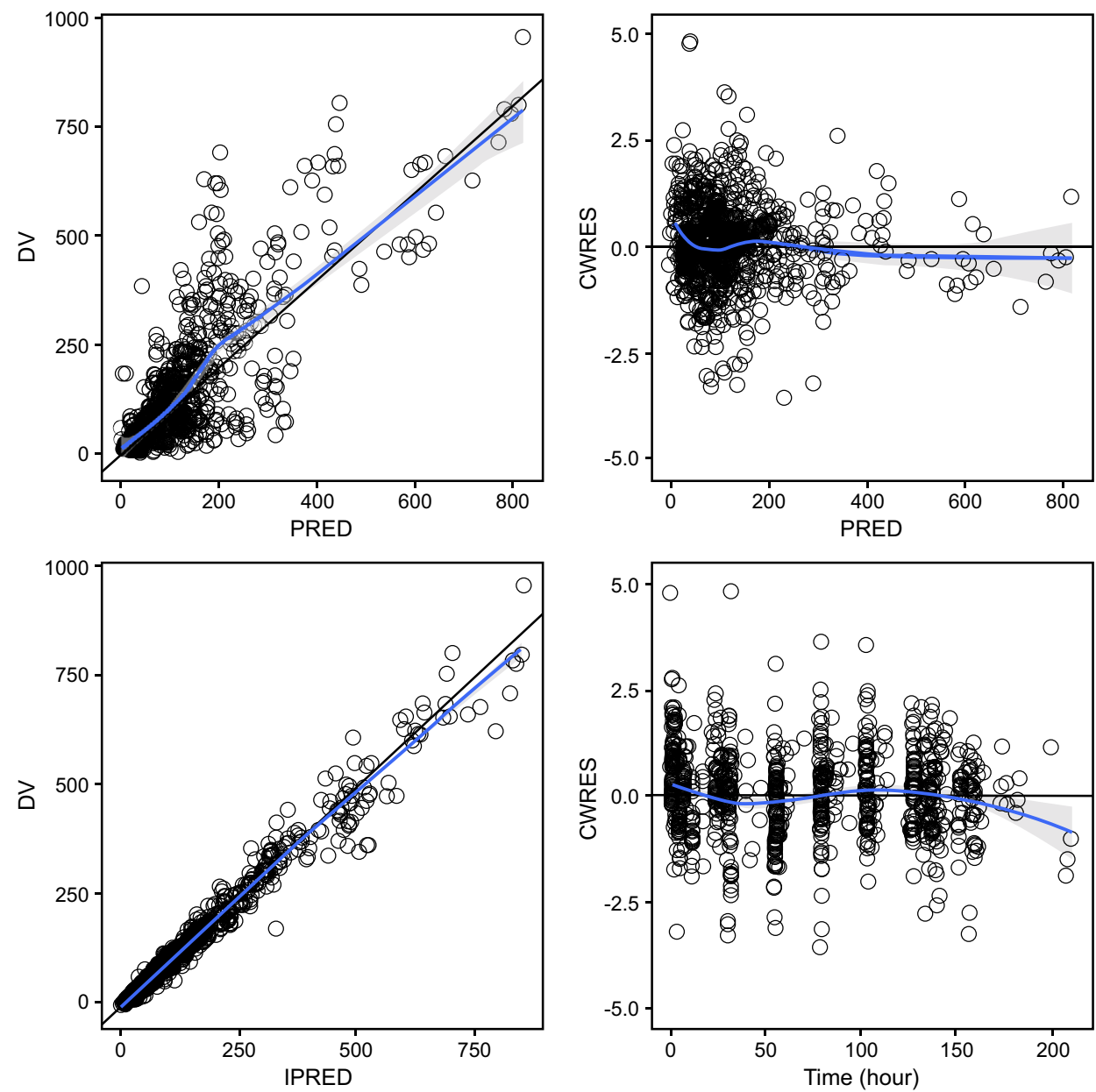

B

Histogram of CWRES

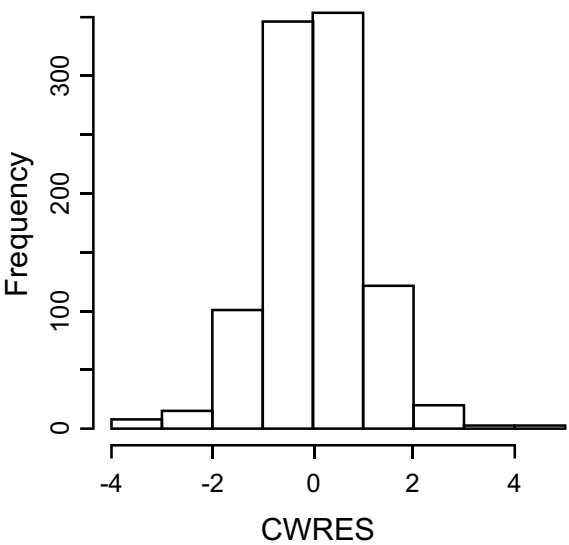

Quantiles of CWRES distribution

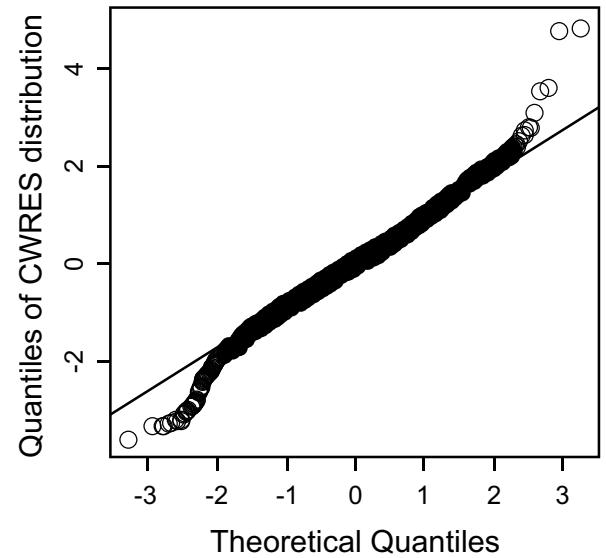

proposed phase III dosing regimen (a 20-g loading dose followed by a 15-g maintenance dose for 5 days for both Caucasian and Asian patients with various degrees of hepatic impairment) and at the reduced dosing regimen (a 15-g loading dose followed by a $10-\mathrm{g}$ maintenance dose for 5 days in
Asian patients with C-P C) are presented in Fig. 5. Because, as already demonstrated, there was no difference between the PK in Chinese and Japanese patients, results here for Asian patients are applicable to Japanese patients. Table 3 
Fig. 4 Visual predictive check for phenylacetic acid (PAA) in patients. $C I$ confidence interval
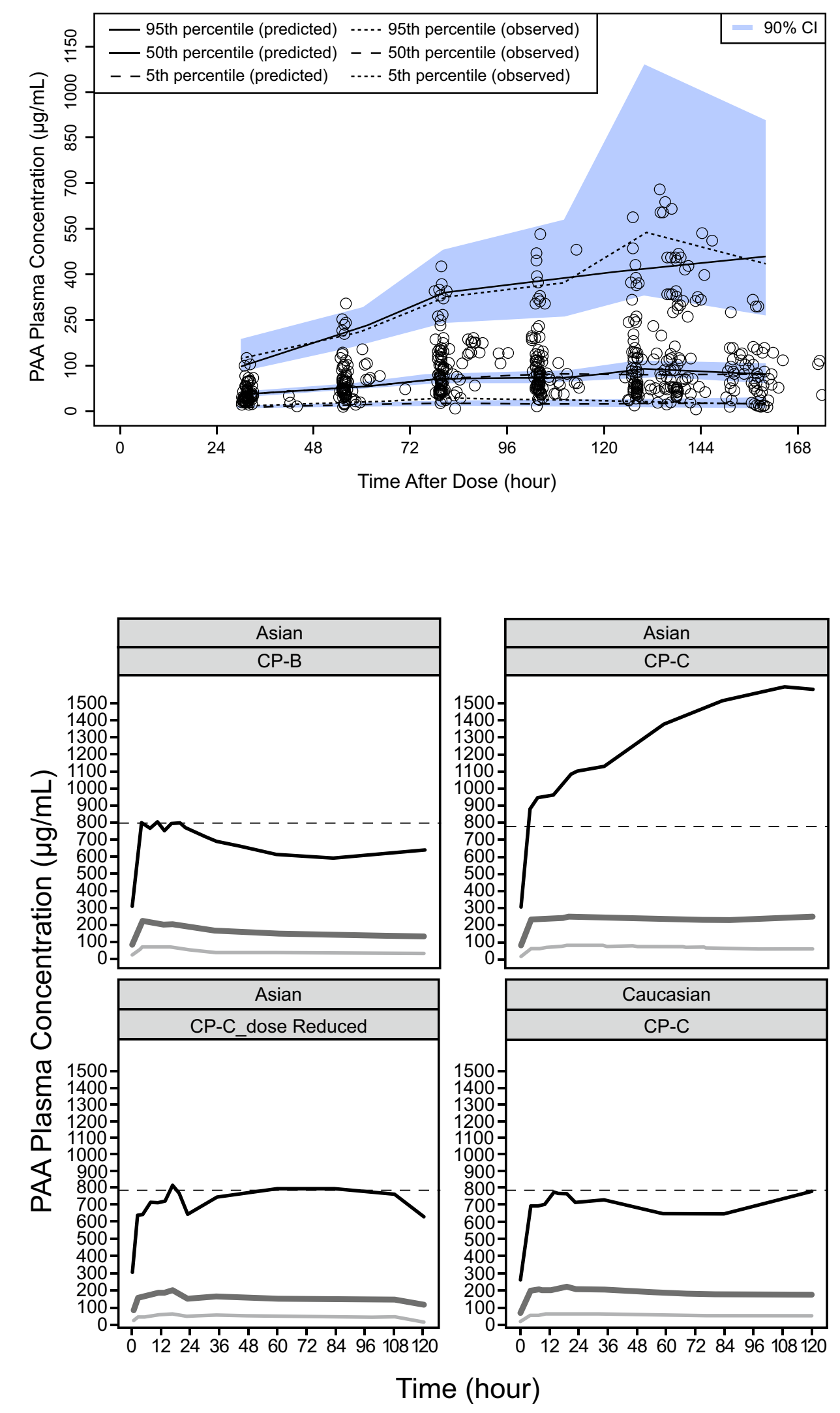

Fig. 5 Simulated phenylacetic acid (PAA) exposure at the phase III dose or at the reduced dosing regimen. $C P-B / C$ Child-Pugh class $\mathrm{B}$ or $\mathrm{C}$. Dosing regimen except for the "CP-C_dose reduced": day $1,20 \mathrm{~g}$ infused over $6 \mathrm{~h}, 15 \mathrm{~g}$ infused over $18 \mathrm{~h}$; day 2 to day 5 , maintenance dose of $15 \mathrm{~g}$ infused over $24 \mathrm{~h}$. CP-C_ dose reduced: day $1,15 \mathrm{~g}$ infused over $6 \mathrm{~h}, 10 \mathrm{~g}$ infused over $18 \mathrm{~h}$; day 2 to day 5 , maintenance dose of $10 \mathrm{~g}$ infused over $24 \mathrm{~h}$. Solid lines: 5th, 50th, and 95th percentiles; dashed line: highest PAA concentration on the 95th percentile for Caucasians with $\mathrm{CP}-\mathrm{C}$ at $20 \mathrm{~g}$ followed by a $15-\mathrm{g}$ maintenance dose 
Table 3 Simulated PAA concentrations in patients

\begin{tabular}{|c|c|c|c|c|c|}
\hline \multirow[t]{2}{*}{ Ethnicity } & \multirow[t]{2}{*}{$\mathrm{C}-\mathrm{P}$ category (dosing regimen ${ }^{\mathrm{a}}$ ) } & \multirow[t]{2}{*}{ Time, $\mathrm{h}$} & \multicolumn{3}{|c|}{ PAA concentrations, $\mu \mathrm{g} / \mathrm{mL}$} \\
\hline & & & Median & 5 th percentile & 95th percentile \\
\hline \multirow[t]{3}{*}{ Caucasian } & \multirow[t]{3}{*}{ C-P B (20 gL_15 gM) } & 84 & 84 & 17 & 369 \\
\hline & & 108 & 88 & 16 & 357 \\
\hline & & 120 & 83 & 16 & 384 \\
\hline \multirow[t]{3}{*}{ Caucasian } & \multirow[t]{3}{*}{ C-P C (20 gL_15 gM) } & 84 & 177 & 52 & 646 \\
\hline & & 108 & 179 & 52 & 730 \\
\hline & & 120 & 175 & 52 & 780 \\
\hline \multirow[t]{3}{*}{ Asian } & \multirow[t]{3}{*}{ C-P B (20 gL_15 gM) } & 84 & 136 & 34 & 578 \\
\hline & & 108 & 129 & 32 & 609 \\
\hline & & 120 & 130 & 30 & 624 \\
\hline \multirow[t]{3}{*}{ Asian } & \multirow[t]{3}{*}{ C-P C (15 gL_10 gM) } & 84 & 144 & 43 & 786 \\
\hline & & 108 & 143 & 43 & 753 \\
\hline & & 120 & 112 & 8 & 622 \\
\hline \multirow[t]{3}{*}{ Asian } & \multirow[t]{3}{*}{ C-P C (20 gL_15 gM) } & 84 & 232 & 66 & 1521 \\
\hline & & 108 & 250 & 61 & 1600 \\
\hline & & 120 & 255 & 63 & 1584 \\
\hline
\end{tabular}

$C-P B / C$ Child-Pugh class $\mathrm{B}$, or $\mathrm{C}, L$ loading dose, $M$ maintenance dose, $P A A$ phenylacetic acid

a $20 \mathrm{gL} \_15 \mathrm{gM}, 20 \mathrm{~g} / 6 \mathrm{~h}$ infusion as a loading dose, $15 \mathrm{~g} / 18 \mathrm{~h}$ infusion, $15 \mathrm{~g} / 24 \mathrm{~h}$ infusion for 4 days; 15 gL_10 gM: $15 \mathrm{~g} / 6 \mathrm{~h}$ infusion as a loading dose, $10 \mathrm{~g} / 18 \mathrm{~h}$ infusion, $10 \mathrm{~g} / 24 \mathrm{~h}$ infusion for 4 days presents the simulated PAA exposure corresponding to the curves in Fig. 5.

Phenylacetic acid exposure in Asian patients with $\mathrm{HE}$ with C-P B was within the ranges of Caucasian patients with $\mathrm{C}-\mathrm{P} \mathrm{C}$, which is expected to be safe based on the outcome of study HE209. However, the 95th percentile of PAA exposure in Asian patients with C-P C was approximately twice as high as that in Caucasian C-P C patients, suggesting that the combination of low body weight and severe hepatic impairment could substantially increase PAA plasma exposure during 5 days of continuous infusion. With a dose reduction to $15 \mathrm{~g}$ as a loading dose and $10 \mathrm{~g}$ as a maintenance dose, the expected PAA exposure for Asian patients with C-P C is comparable to Caucasian patients with $\mathrm{HE}$ with $\mathrm{C}-\mathrm{P} \mathrm{C}$.

\section{Discussion}

Our analysis demonstrated that the two most important factors that determine the exposure to PAA are body weight and hepatic dysfunction. No statistical difference was observed between Caucasian and Asian patients once body weight was taken into consideration. Body size (expressed using either body weight or BSA) has been reported to have significant impact on PAA exposure in patients with UCD [7]. The approved doses of sodium phenylbutyrate and glycerol phenylbutyrate for UCD are based on body weight or BSA [4-6]. In Japanese patients with UCD, sodium phenylbutyrate follows the same dosing algorithm as in Caucasian patients, suggesting that ethnicity does not play a role in a weight-based or BSA-based dosing algorithm [9].

Patients with hepatic dysfunction may have other underlying disease that can contribute to the development of HE. Renal dysfunction can be observed in patients with HE. Elevated plasma concentration of PAA was expected in patients based on the severity of hepatic impairment. Population PK modeling identified that PAA exposure was approximately $36 \%$ higher in patients with $\mathrm{C}-\mathrm{P} \mathrm{C}$ than in patients with $\mathrm{C}-\mathrm{P} \mathrm{B}$. This derivation agreed with the observed PAA concentration at steady state from study HE209, where C-P C patients had a $35 \%$ higher exposure to PAA versus C-P B patients [8].

Renal dysfunction has no impact on plasma concentration of PAA [19]. That renal dysfunction does not alter PAA exposure confirmed the hypothesis that the formation of PAGN is an irreversible process, thus the accumulation of PAGN in blood circulation due to reduced renal excretion would not alter the rate of PAGN formation. As such, renal dysfunction is not expected to change the removal of free ammonia in plasma circulation either, as observed from study HE209 [8]. To confirm this derivation, the present population PK model was applied to the PK data from a newly completed renal impairment study that included 
patients with severe renal impairment. The change in the objective function was $<0.2$ point, confirming that renal function was not a statistically significant covariate for PAA plasma concentration (unpublished data, Mallinckrodt Pharmaceuticals).

The combination of low body weight and severe hepatic impairment could result in a substantial increase in PAA exposure owing to its nonlinear biotransformation. The originally proposed phase III dose for Caucasian patients is not based on weight or BSA, nor is adjustment for liver function required. This flat dose of $15 \mathrm{~g}$ for Caucasians was supported by the safety and efficacy data from the HE209 study (NCT01966419) mentioned previously. However, Asian individuals in general have a lower body weight. When individuals have both a low body weight and severe hepatic impairment, an over-proportional increase in PAA with multiple doses could put them at serious risk. Although this scenario is less likely to happen in Caucasian patients, attention needs to be paid to patients with a low body weight and severe hepatic impairment. Model-based simulation suggests that dose reduction, from 20 to $15 \mathrm{~g}$ for the loading dose and from 15 to $10 \mathrm{~g}$ for the maintenance dose, for Asian patients with C-P C can maintain PAA exposure within the range that is clinically proven to be safe. After further discussion with health authorities, all patients (both Caucasian and Asian) with a body weight at $50 \mathrm{~kg}$ and below will be administered the reduced-dose level. Simulation of PAA exposure at two dose levels with a body weight cut-off of $50 \mathrm{~kg}$ is presented in Fig. S6 of the ESM, and confirmed that the reduced-dose level can ensure PAA exposure below the observed level from the phase IIb study.

The administration of ORN to increase the formation of PAGN, which also enhanced ammonia elimination, was studied in an exploratory analysis. The estimated coefficient in the expression of Coe $\times C_{\mathrm{ORN}}$ was 1.22 . At $15 \mathrm{~g}$ of L-OPA, the observed median concentration of ORN (including both endogenous and exogenous) was about $30 \mu \mathrm{g} / \mathrm{mL}$. The endogenous concentration of ORN is approximately $10 \mu \mathrm{g} / \mathrm{mL}$ [21]. Therefore, ORN exposure from the administration of L-OPA was in the range of $20 \mu \mathrm{g} / \mathrm{mL}(0.15 \mu \mathrm{mol} / \mathrm{mL})$. The estimated $V_{\max }$ increase was approximately $18 \%$. Because L-OPA has a fixed 1:1 molar ratio of ORN:PAA, to accurately estimate enhancement in $V_{\max }$ with the administration of ORN would require that different ratios of ORN:PAA be administered.

Last, the advantages of the simplified model over the model depicted in Fig. 2 were not only in the computation time but also in the performance of data description. The computation time for 1 NONMEM execution of the simplified model was about $10 \%$ that of the model shown in Fig. 2. The simplified model adequately described the observed plasma concentrations of ORN, PAA, and PAGN without bias. Results derived from the covariate analysis using the simplified model also agreed with the observed clinical data or published information (literature-based or PAA-based drug label).

In summary, both body weight and hepatic impairment were the most important covariates for PAA exposure. After accounting for body weight, there was no difference in PAA exposure between Caucasian and Asian populations. However, when a flat dosing algorithm is adopted for L-OPA, special considerations such as dose reduction must be given to patients with a small body size and severe hepatic impairment, regardless of ethnicity. Although renal impairment significantly reduces PAGN clearance, renal impairment has no impact on the biotransformation of PAA, as well as the reduction in the free ammonia level in plasma circulation.

Supplementary Information The online version contains supplementary material available at https://doi.org/10.1007/s40262-021-01075-1.

Acknowledgements Financial support was provided by Mallinckrodt Pharmaceuticals. Editorial support for preparation of this manuscript was provided by Peloton Advantage, LLC, an OPEN Health company, Parsippany, NJ, USA, and funded by Mallinckrodt Pharmaceuticals. The authors thank Greg Tardie, PhD of Mallinckrodt Pharmaceuticals for his scientific and editorial contributions to the draft.

\section{Declarations}

Funding This study was sponsored by Mallinckrodt Pharmaceuticals, which participated in the review of and decision to publish this manuscript.

Conflict of interest Xiaofeng Wang is a former employee of Mallinckrodt Pharmaceuticals. Regis A. Vilchez is a current employee of Mallinckrodt Pharmaceuticals. The study sponsor was involved in the study design and data collection, analysis, and interpretation.

Ethics approval An institutional review board approved each study protocol.

Consent to participate All participants gave written informed consent prior to the initiation of study procedures.

Consent for publication Not applicable (no personal data were reported).

Availability of data and material Discussion of statistical endpoints and analysis are included in the manuscript. Summary aggregate (basic) results (including adverse event information) and the protocol for study HE209 will be available on clinicaltrials.gov (NCT01966419) when required by regulation. Individual de-identified patient data will not be disclosed. Requests for additional information should be directed to the company at medinfo@mnk.com.

Code availability NONMEM (version 7.4, Icon Development Solutions, Ellicott City, MD, USA) and R (version 3.5.2.).

Author contributions XW drafted the manuscript; conducted pharmacokinetics and exposure-adverse event analyses, and interpreted the outcome of the analyses included in the manuscript; was a key contributor to the design of the two clinical studies; and approved the manuscript. RAV designed and analyzed the study, and reviewed and approved the manuscript. 
Previous publication None.

Open Access This article is licensed under a Creative Commons Attribution-NonCommercial 4.0 International License, which permits any non-commercial use, sharing, adaptation, distribution and reproduction in any medium or format, as long as you give appropriate credit to the original author(s) and the source, provide a link to the Creative Commons licence, and indicate if changes were made. The images or other third party material in this article are included in the article's Creative Commons licence, unless indicated otherwise in a credit line to the material. If material is not included in the article's Creative Commons licence and your intended use is not permitted by statutory regulation or exceeds the permitted use, you will need to obtain permission directly from the copyright holder. To view a copy of this licence, visit http://creativecommons.org/licenses/by-nc/4.0/.

\section{References}

1. Vilstrup H, Amodio P, Bajaj J, et al. Hepatic encephalopathy in chronic liver disease: 2014 practice guideline by the American Association for the Study of Liver Diseases and the European Association for the Study of the Liver. Hepatology. 2014;60:715-35.

2. Wijdicks EF. Hepatic encephalopathy. N Engl J Med. 2016;375:1660-70.

3. De Las HJ, Aldámiz-Echevarría L, Martínez-Chantar ML, Delgado TC. An update on the use of benzoate, phenylacetate and phenylbutyrate ammonia scavengers for interrogating and modifying liver nitrogen metabolism and its implications in urea cycle disorders and liver disease. Expert Opin Drug Metab Toxicol. 2017;13:439-48.

4. Buphenyl [package insert]. Scottsdale: Ucyclyd Pharma, Inc.; 2008.

5. Ravicti [package insert]. Lake Forest: Horizon Pharma USA, Inc.; 2019.

6. Ravicti [summary of product characteristics]. Sweden: Immedica Pharma; 2020.

7. Monteleone JP, Mokhtarani M, Diaz GA, et al. Population pharmacokinetic modeling and dosing simulations of nitrogen-scavenging compounds: disposition of glycerol phenylbutyrate and sodium phenylbutyrate in adult and pediatric patients with urea cycle disorders. J Clin Pharmacol. 2013;53:699-710.

8. Wang X, Tseng J, Mak C, Poola N, Vilchez RA. Exposures of phenylacetic acid and phenylacetylglutamine across different subpopulations and correlation with adverse events. Clin Pharmacokinet. 2021. https://doi.org/10.1007/s40262-021-01047-5 (Epub ahead of print).
9. Buphenyl [Japanese package insert]. Tokyo: Orphan Pacific DI Center Co., Ltd.; 2015.

10. Jalan R, Wright G, Davies NA, Hodges SJ. L-Ornithine phenylacetate (OP): a novel treatment for hyperammonemia and hepatic encephalopathy. Med Hypotheses. 2007;69:1064-9.

11. Thibault A, Samid D, Cooper MR, et al. Phase I study of phenylacetate administered twice daily to patients with cancer. Cancer. 1995;75:2932-8.

12. Rahimi RS, Safadi R, Thabut D, et al. Efficacy and safety of ornithine phenylacetate for treating overt hepatic encephalopathy in a randomized trial. Clin Gastroenterol Hepatol. 2020. (Epub ahead of print).

13. Hung TH, Tseng CW, Tseng KC, Hsieh YH, Tsai CC, Tsai CC. Effect of renal function impairment on the mortality of cirrhotic patients with hepatic encephalopathy: a population-based 3-year follow-up study. Medicine. 2014;93:e79.

14. Aggarwal HK, Jain D, Singla S, Jain P. Assessment of renal functions in patients of chronic liver disease. Ren Fail. 2015;37:1457-63.

15. Office of Minority Health Resource Center. Obesity and Asian Americans. 2020. Available from: https://minorityhealth.hhs.gov/ omh/browse. aspx ?lvl=4\&lvlid=55. Accessed 6 Nov 2020.

16. Ramachandran A, Chamukuttan S, Shetty SA, Arun N, Susairaj P. Obesity in Asia: is it different from rest of the world. Diabetes Metab Res Rev. 2012;28(Suppl. 2):47-51.

17. Anderson K, Fischer L, Hassanein T, Kittrelle J, Cato J. Randomized, double-blind, placebo-controlled, ascending dose (SAD/ MAD) studies to evaluate the safety and pharmacokinetics of the ammonia scavenger OCR-002 (ornithine phenylacetate) in healthy volunteers (HV) and patients with stable hepatic cirrhosis [abstract PP37-018]. Hepatol Int. 2012;6:297-8.

18. Hassanein T, Kittrelle J, Cato A, Fischer L, Anderson K. A randomized, double-blind, placebo-controlled, single ascending dose study to evaluate the safety and pharmacokinetics of OCR-022 (ornithine phenylacetate) in patients with stable hepatic cirrhosis [abstract 1210]. Hepatology. 2011;54(4 Suppl.):934A-A935.

19. Stravitz RT, Gottfried M, Durkalski V, et al. Safety, tolerability, and pharmacokinetics of L-ornithine phenylacetate in patients with acute liver injury/failure and hyperammonemia. Hepatology. 2018;67:1003-13.

20. Kircheis G, Lüth S. Pharmacokinetic and pharmacodynamic properties of L-ornithine L-aspartate (LOLA) in hepatic encephalopathy. Drugs. 2019;79:23-9.

21. Lai X, Kline JA, Wang M. Development, validation, and comparison of four methods to simultaneously quantify L-arginine, citrulline, and ornithine in human plasma using hydrophilic interaction liquid chromatography and electrospray tandem mass spectrometry. J Chromatogr B Analyt Technol Biomed Life Sci. 2015;1005:47-55. 